SU-ITP-99/27

KUL-TF-99/21

hep-th/9906118

June 15, 1999

\title{
A Simple Particle Action from a Twistor Parametrization of $\mathrm{AdS}_{5}$
}

\author{
Piet Claus $^{\dagger a}$, J. Rahmfeld ${ }^{* b}$ and Yonatan Zunger ${ }^{* c}$ \\ $\dagger$ Instituut voor theoretische fysica, \\ Katholieke Universiteit Leuven, B-3001 Leuven, Belgium \\ * Physics Department, \\ Stanford University, Stanford, CA 94305-4060, USA
}

\begin{abstract}
The $S O(4,2)$ isometries of $A d S_{5}$ are realized non-linearly on its horospherical coordinates $\left(x^{m}, \rho\right)$. On the other hand, Penrose twistors have long been known to linearly realize these symmetries on 4-dimensional Minkowski space, the boundary of $A d S_{5}$, parametrized by $x^{m}$. Here we extend the twistor construction and define a pair of twistors, allowing us to include a radial coordinate in the construction. The linear action of $S O(4,2)$ on the twistors induces the correct isometries of $A d S_{5}$. We apply this new construction to the study of the dynamics of a massive particle in $A d S_{5}$. We show that in terms of the twistor variables the action takes a simple form of a 1-dimensional gauge theory. Our result might open up the possibility to find a simple worldvolume action also for the string propagating on $A d S_{5}$.
\end{abstract}

\footnotetext{
${ }^{a}$ e-mail: piet.claus@fys.kuleuven.ac.be.

${ }^{b}$ e-mail: rahmfeld@leland.stanford.edu.

${ }^{c}$ e-mail: zunger@leland.stanford.edu.
} 
In [1] a two-dimensional world-sheet model was considered with manifest $S U(2,2 \mid 4)$ symmetry. The basic variables of the model are supertwistors which realize the symmetry linearly. From the supertwistors one can derive $4 x^{m}$ and $16 \theta$ [2, 3] coordinates on which $S U(2,2 \mid 4)$ is realized as the non-linear superconformal symmetries. Since the same symmetry group also appears as the isometry group of the $A d S_{5} \times S^{5}$ superspace, it is natural to wonder in view of the $A d S / \mathrm{CFT}$ correspondence [1], whether supertwistors might be useful variables to describe strings in this background. In this brief note, we extend the purely bosonic twistor construction to include a radial coordinate, based on the coset construction of $A d S_{5}$. The linear action of the $\operatorname{SU}(2,2)$ group on these twistors induces the correct isometries of $A d S_{5}$. As a warm-up to applying these new variables to strings propagating in an $A d S_{5} \times S^{5}$ superspace, we then consider the (non-linear) action of a massive particle on $A d S_{5}$ and show how the new twistors bring the action into a simple (and quantizable |50) form, generalizing the work of [6]. In fact, the resulting theory is nothing but a one-dimensional $U(1) \times S U(2)$ gauge theory of the form

$$
S=-i \int d \tau\left(\overline{\mathcal{Z}}^{I} D_{\tau} \mathcal{Z}^{I}+2 i u^{0} m R\right)
$$

where

$$
D_{\tau} \mathcal{Z}^{I}=\left(\delta^{I J} \partial_{\tau}-i u^{a} t_{a}^{I J}\right) \mathcal{Z}^{J}
$$

and the $t_{a}^{I J}$ matrices are $U(1) \times S U(2)$ generators. The rest of the notation will be explained in the main body.

We begin by recalling the $A d S_{5}$ isometries, their relation to the standard twistor formalism in the boundary limit, and the general construction of coset isometries. $A d S_{5}$ space in horospherical coordinates $\tilde{x}^{\tilde{m}}=\left(x^{m}, \rho\right)$ is described by the metric

$$
d s^{2}=d \tilde{x}^{\tilde{m}} \tilde{g}_{\tilde{m} \tilde{n}} d \tilde{x}^{\tilde{n}}=e^{m} \eta_{m n} e^{n}+e^{\rho} e^{\rho}=\rho^{2} d x^{2}+R^{2}\left(\frac{d \rho}{\rho}\right)^{2}
$$

where $R$ is the radius of curvature. The isometries and compensating Lorentz transformations $\ell$ (acting on $e^{m}, e^{\rho}$ ) [7] are given by

$$
\begin{aligned}
\delta x^{m} & =-\left(a^{m}+\lambda_{M}^{m n} x_{n}+\lambda_{D} x^{m}+x^{2} \Lambda_{K}^{m}-2 x \cdot \Lambda_{K} x^{m}\right)-R^{2}\left(\frac{\Lambda_{K}^{m}}{\rho^{2}}\right), \\
\delta \rho & =\left(\lambda_{D}-2 x \cdot \Lambda_{K}\right) \rho, \\
\ell^{m n}(\tilde{x}) & =\lambda_{M}^{m n}-4 x^{[m} \Lambda_{K}^{n]}, \\
\ell^{m \rho}(\tilde{x}) & =-2 R \frac{\Lambda_{K}^{m}}{\rho},
\end{aligned}
$$

where $a^{m}, \lambda_{M}^{m n}, \lambda_{D}$, and $\Lambda_{K}^{m}$ are the constant parameters of translations, Lorentz boosts, dilatation, and special conformal transformations. 
In the limit $\rho \rightarrow \infty$ the radial coordinate decouples from the transformations and one is left with the conformal transformations of 4-dimensional Minkowski space. These non-linear transformations are nicely encoded in the transformation properties of a twistor円

$$
\mathcal{Z} \equiv\left(\begin{array}{c}
\lambda_{\alpha} \\
\bar{\mu}^{\dot{\alpha}}
\end{array}\right)
$$

transforming under rigid transformations $g$ in the fundamental of $S U(2,2) \mathbb{f}$

$$
\delta\left(\begin{array}{c}
\lambda_{\alpha} \\
\bar{\mu}^{\dot{\alpha}}
\end{array}\right)=-g\left(\begin{array}{c}
\lambda_{\alpha} \\
\bar{\mu}^{\dot{\alpha}}
\end{array}\right) \equiv\left(\begin{array}{cc}
L_{\alpha}^{\beta}+\frac{1}{2} D \delta_{\alpha}^{\beta} & i K_{\alpha \dot{\beta}} \\
i A^{\dot{\alpha} \beta} & -\bar{L}_{\dot{\beta}}^{\dot{\alpha}}-\frac{1}{2} D \delta_{\dot{\beta}}^{\dot{\alpha}}
\end{array}\right)\left(\begin{array}{c}
\lambda_{\beta} \\
\bar{\mu}^{\dot{\beta}}
\end{array}\right),
$$

where we used the spinorial notation (see the appendix)

$$
\begin{aligned}
x^{\dot{\alpha} \alpha} & =\frac{x^{m}}{R}\left(\bar{\sigma}_{m}\right)^{\dot{\alpha} \alpha}, \\
A^{\dot{\alpha} \alpha} & =\frac{a^{m}}{R}\left(\bar{\sigma}_{m}\right)^{\dot{\alpha} \alpha}, \quad K_{\alpha \dot{\alpha}}=R \Lambda_{K}^{m}\left(\sigma_{m}\right)_{\alpha \dot{\alpha}}, \quad D=\lambda_{D}, \\
L_{\alpha}^{\beta} & =\frac{1}{4} \lambda_{M}^{m n}\left(\sigma_{m n}\right)_{\alpha}{ }^{\beta}, \quad \bar{L}_{\dot{\beta}}^{\dot{\alpha}}=-\frac{1}{4} \lambda_{M}^{m n}\left(\bar{\sigma}_{m n}\right)_{\dot{\beta}}^{\dot{\alpha}},
\end{aligned}
$$

i.e. we choose to take all our variables dimensionless. The conformal twistor relation between the two spinors $\lambda$ and $\bar{\mu}$,

$$
\bar{\mu}^{\dot{\alpha}}=-i x^{\dot{\alpha} \alpha} \lambda_{\alpha}
$$

then induces the non-linear conformal transformations of $x$ from the simple linear transformation of the twistor $\mathcal{Z}$. The non-trivial step in this parametrization of conformal space is the twistor relation (8), which can be derived from the dynamics of a massless particle [3]. However, here we derive the twistor relation from a purely algebraic perspective, as the twistor parametrization of conformal space is closely related to the coset-space construction. This will allow us to generalize the procedure to the full $A d S_{5}$ space.

The parametrization of a coset space is encoded in the coset representative $v(X)$, where $X$ are the coordinates of the space. As a function of coordinates $v(X)$ transforms under an infinitesimal action of the group $G$ as

$$
\delta v(X) \equiv-\xi_{g}^{m}(X) \partial_{m} v(X)=-g v(X)+v(X) h_{g}(X)
$$

with $m=1, \ldots, \operatorname{dim} G / H, g \in \mathbf{G}$, the Lie-algebra associated to $G$, and $h_{g}(X)$ is a space-time dependent compensating transformation in the Lie-algebra of $H$. Eq. (9) is equivalent to the general relation [7]

$$
v^{-1} g v=\xi_{g}^{m} L_{m}+h_{g}(X),
$$

\footnotetext{
${ }^{1}$ As usual in the twistor literature, $\lambda_{\alpha}$ and $\bar{\mu}^{\dot{\alpha}}$ are commuting, two-component complex spinors. Our conventions are explained in the appendix.

${ }^{2}$ The universal covering group of the conformal group $S O(4,2)$ is $S U(2,2)$. Its compact $S U(2)$ subgroups act as rotations on chiral and antichiral spinors, respectively.
} 
where $L_{m}=v^{-1} \partial_{m} v$ is the Cartan 1 -form in $\mathbf{G}$. The $\xi_{g}^{m}$ are the isometries, $\delta X^{m}=$ $-\xi_{g}^{m}(X)$, that realize $G$ non-linearly on the coordinates. Clearly, an object

$$
\mathcal{Z}=v(X) \mathcal{Z}_{0}
$$

will transform rigidly in the fundamental of $G$, i.e. $\delta \mathcal{Z}=-g \mathcal{Z}$, iff $\delta \mathcal{Z}_{0}=-h_{g}(X) \mathcal{Z}_{0}$.

Twistors turn this reasoning upside down. Whenever we define a vector $\mathcal{Z}_{0}$ by (11) and demand that $\mathcal{Z}$ transforms in the fundamental of $G$, it is guaranteed that $\mathcal{Z}_{0}$ will transform under the correct compensating transformation $h_{g}(X)$. If we want to impose a twistor relation on $\mathcal{Z}$, it turns out to be most useful to put a constraint on $\mathcal{Z}_{0}$, effectively relating the components of $\mathcal{Z}$ via $X$. Hereby, one only has to demand that the constraint is covariant under the compensating $H$-transformations. A consistent twistor relation can therefore be obtained from the coset space by defining a vector $\mathcal{Z}_{0}$ through (11) and constraining it in a $H$ covariant way.

We illustrate the above discussion with the twistor formulation of conformal space. The conformal transformations in spinorial notation

$$
\delta x^{\dot{\alpha} \alpha}=-\left(A^{\dot{\alpha} \alpha}+x^{\dot{\alpha} \beta} L_{\beta}^{\alpha}+\bar{L}_{\dot{\beta}}^{\dot{\alpha}} x^{\dot{\beta} \alpha}+D x^{\dot{\alpha} \alpha}+x^{\dot{\alpha} \beta} K_{\beta \dot{\beta}} x^{\dot{\beta} \alpha}\right),
$$

induces the compensating $H$ transformation

$$
h_{g}^{\text {Conf }}=\left(\begin{array}{cc}
-L_{\alpha}{ }^{\beta}-\frac{D}{2} \delta_{\alpha}{ }^{\beta}-K_{\alpha \dot{\alpha}} x^{\dot{\alpha} \beta} & -i K_{\alpha \dot{\alpha}} \\
0 & \bar{L}_{\dot{\beta}}^{\dot{\alpha}}+\frac{D}{2} \delta_{\dot{\beta}}^{\dot{\alpha}}{ }^{\dot{\alpha} \beta} K_{\beta \dot{\beta}}
\end{array}\right) .
$$

These transformations are simply the isometries of the coset space $S U(2,2) / I S O(1,3) \times$ $D$, with the coset representative

$$
v^{C o n f}(x)=\left(\begin{array}{cc}
\delta_{\alpha}{ }^{\beta} & 0 \\
-i x^{\dot{\alpha} \alpha} & \delta^{\dot{\alpha}}{ }_{\dot{\beta}}
\end{array}\right)
$$

From (5) and (11) one constructs

$$
\mathcal{Z}_{0} \equiv\left(\begin{array}{c}
\lambda_{0 \alpha} \\
\bar{\mu}_{0}^{\dot{\alpha}}
\end{array}\right)=\left(\begin{array}{c}
\lambda_{\alpha} \\
i x^{\dot{\alpha} \alpha} \lambda_{\alpha}+\bar{\mu}^{\dot{\alpha}}
\end{array}\right)
$$

which needs to be constrained $H$-covariantly. The only form of $\mathcal{Z}_{0}$ transforming covariantly under (13) is

$$
\mathcal{Z}_{0}=\left(\begin{array}{c}
\lambda_{0 \alpha} \\
0
\end{array}\right)
$$

which implies the conformal twistor relation (8), as desired. In this case finding the proper constraint (16) was straightforward. The $S O(1,3)$ in $H$ allows for chiral/antichiral spinors, or Majorana spinors. However, only the chiral spinor $\lambda_{0}^{\alpha}$ of (16) transforms covariantly under special conformal transformations. 
This example illustrates nicely the method we have developed of constructing twistors via the coset representative. We can now extend the procedure to the full $A d S_{5}$ case.

The metric and isometries (3) and (4) follow from the coset construction of $A d S_{5}=S O(4,2) / S O(4,1)$. We choose the coset representative $v^{A d S}\left(x^{m}, \rho\right)$ in the spinor representation of $S O(4,2)$

$$
v^{A d S}(x, \rho)=\left(\begin{array}{cc}
\rho^{1 / 2} \delta_{\alpha}{ }^{\beta} & 0 \\
-i \rho^{1 / 2} x^{\dot{\alpha} \alpha} & \rho^{-1 / 2} \delta_{\dot{\beta}}^{\dot{\alpha}}
\end{array}\right)
$$

and, in spinorial notation, the isometries (4) take the form

$$
\begin{aligned}
\delta x^{\dot{\alpha} \alpha} & =-\left(A^{\dot{\alpha} \alpha}+x^{\dot{\alpha} \beta} L_{\beta}{ }^{\alpha}+\bar{L}_{\dot{\beta}}^{\dot{\alpha}} x^{\dot{\beta} \alpha}+D x^{\dot{\alpha} \alpha}+x^{\dot{\alpha} \beta} K_{\beta \dot{\beta}} x^{\dot{\beta} \alpha}\right)-K^{\dot{\alpha} \alpha} \rho^{-2}, \\
\delta \rho & =\left(D+K^{\dot{\alpha} \alpha} x_{\alpha \dot{\alpha}}\right) \rho .
\end{aligned}
$$

The compensating $S O(1,4)$ transformation reads

$$
h_{g}^{A d S}=\left(\begin{array}{cc}
-L_{\alpha}^{\beta}-K_{\alpha \dot{\alpha}} x^{\dot{\alpha} \beta}+\frac{1}{2} K_{\gamma \dot{\gamma}} x^{\dot{\gamma} \gamma} \delta_{\alpha}^{\beta} & -i \rho^{-1} K_{\alpha \dot{\beta}} \\
i \rho^{-1} K^{\dot{\alpha} \beta} & \bar{L}_{\dot{\beta}}^{\dot{\alpha}}-x^{\dot{\alpha} \beta} K_{\beta \dot{\beta}}+\frac{1}{2} x^{\dot{\gamma} \gamma} K_{\gamma \dot{\gamma}} \delta_{\dot{\beta}}^{\dot{\alpha}}
\end{array}\right) .
$$

We wish to replace the coordinates $\left(x^{m}, \rho\right)$ with twistor variables, on which a linear $S U(2,2)$ transformation induces (18) and (19). Naturally, one would want to define as above a twistor $\mathcal{Z}$, and relate it via the coset representative to a vector $\mathcal{Z}_{0}$. On $\mathcal{Z}_{0}$ then a constraint has to be imposed transforming covariantly under $H$, effectively relating the components of $\mathcal{Z}$ via the coset coordinates. In this case we have $H=S O(1,4)$, and $\mathcal{Z}_{0}$ should transform in the spinor representation of this group. There are neither Weyl nor Majorana fermions in $(1,4)$ dimensions, and there does not exist a covariantly constrained spinor. Therefore, a straightforward extension of (8) incorporating the radial $\rho$ coordinate of the $A d S$ space does not exist. However, in $(1,4)$ dimensions one can define pseudo-symplectic Majorana fermions $\psi^{I}$ satisfying

$$
\left(\psi^{I}\right)^{*}=\epsilon^{I J} B \psi^{J}, \quad I, J=1,2 .
$$

The matrix $B$ satisfies

$$
B^{T}=-B, \quad B^{\dagger} B=1, \quad B B^{*}=-1, \quad \gamma_{\tilde{m}}^{*}=-B \gamma_{\tilde{m}} B^{-1},
$$

where $\gamma_{\tilde{m}}$ are the 5 -dimensional $\gamma$-matrices. In the two-component notation used above the matrix $B$ takes the form

$$
B=\left(\begin{array}{cc}
0 & -\varepsilon_{\dot{\alpha} \dot{\beta}} \\
\varepsilon^{\alpha \beta} & 0
\end{array}\right) .
$$


Since one can impose a restriction only on a pair of spinors, we have to extend the twistor construction to a pair of twistors

$$
\mathcal{Z}^{I}=\left(\begin{array}{c}
\lambda_{\alpha}^{I} \\
\bar{\mu}^{\dot{\alpha} I}
\end{array}\right)
$$

transforming in the fundamental of $S U(2,2)$

$$
\delta \mathcal{Z}^{I}=-g \mathcal{Z}^{I}
$$

Just as in (11) one relates these twistors to a pair of spinors $\mathcal{Z}_{0}^{I}$ via

$$
\mathcal{Z}^{I}=v^{A d S}(x, \rho) \mathcal{Z}_{0}^{I}
$$

where $\mathcal{Z}_{0}^{I}$ transforms as

$$
\delta \mathcal{Z}_{0}^{I}=-h_{g}(X) \mathcal{Z}_{0}^{I}
$$

As discussed, the only $H$-covariant condition which can be placed on the $\mathcal{Z}_{0}^{I}$ is the pseudo-symplectic Majorana condition

$$
\left(\mathcal{Z}_{0}^{I}\right)^{*}=\epsilon^{I J} B \mathcal{Z}_{0}^{J}
$$

which leads to the form

$$
\mathcal{Z}_{0}^{I}=\left(\begin{array}{c}
\lambda_{0 \alpha}^{I} \\
\epsilon^{I J} \bar{\lambda}_{0}^{\dot{\alpha} J}
\end{array}\right)
$$

with unrestricted $\lambda_{0}^{I}$ and $\lambda_{0}^{\dot{\alpha} I}=\left(\lambda_{0 \beta}^{I}\right)^{*} \varepsilon^{\dot{\beta} \dot{\alpha}}$. The generalization of (8) now becomes

$$
\bar{\mu}^{\dot{\alpha} I}=-i x^{\dot{\alpha} \alpha} \lambda_{\alpha}^{I}+\frac{\epsilon^{I J}}{\rho} \bar{\lambda}^{\dot{\alpha} J} .
$$

It is straightforward to verify that from this $A d S$ twistor relation, (24) and (6), the AdS isometries (18) follow.

The pair of twistors trade the 5 coordinates $\left(x^{m}, \rho\right)$ for 8 complex degrees of freedom $\mathcal{Z}^{I}$, which are related as in (29). This relation was derived from a purely algebraic perspective. Using this explicit relation we can find the invariant quadratic constraints the twistor pair satisfies. The $S U(2,2)$ group can be defined as the transformations that leave the metric

$$
H_{S U(2,2)}=\left(\begin{array}{ll}
0 & \mathbb{1} \\
\mathbb{1} & 0
\end{array}\right)
$$

invariant. Therefore the bilinear form

$$
\overline{\mathcal{Z}}(1) \mathcal{Z}(2)=\mu^{\alpha}(1) \lambda_{\alpha}(2)+\bar{\lambda}_{\dot{\alpha}}(1) \bar{\mu}^{\dot{\alpha}}(2)
$$


where

$$
\overline{\mathcal{Z}}=\mathcal{Z}^{\dagger} H=\left(\mu^{\alpha}, \bar{\lambda}_{\dot{\alpha}}\right)
$$

is the conjugate representation, is a quadratic invariant. With a pair of twistors we can define the general bilinear invariant

$$
\mathcal{Z}^{I} g^{I J} \mathcal{Z}^{J}=\mathcal{Z}_{0}^{I} g^{I J} \mathcal{Z}_{0}^{J}
$$

where $g^{I J}$ is 2-dimensional constant complex matrix. The equality above holds because $\mathcal{Z}^{I}$ and $\mathcal{Z}_{0}^{I}$ are related by the $S U(2,2)$ matrix $v^{A d S}$. We can expand the matrix $g$ in a basis

$$
t_{a}^{I J}=\left\{\delta^{I J},\left(\hat{\sigma}_{i}\right)^{I J}\right\},
$$

where $\hat{\sigma}_{i}$ are the Pauli-matrices satisfying $\hat{\sigma}_{1} \hat{\sigma}_{2}=i \hat{\sigma}_{3}$ and $a$ takes values $0,1,2,3$. From the relation (29) we obtain the constraints

$$
\overline{\mathcal{Z}}^{I} \hat{\sigma}_{i}^{I J} \mathcal{Z}^{J}=0
$$

which imply that the two components $\mathcal{Z}^{1}$ and $\mathcal{Z}^{2}$ of the twistor pair have the same 'length' ' $\overline{\mathcal{Z}}^{1} \mathcal{Z}^{1}=\overline{\mathcal{Z}}^{2} \mathcal{Z}^{2}$ and are 'orthogonal' $\overline{\mathcal{Z}}^{1} \mathcal{Z}^{2}=\overline{\mathcal{Z}}^{2} \mathcal{Z}^{1}=0$. However, there is a fourth independent invariant bilinear

$$
\overline{\mathcal{Z}}^{I} \delta^{I J} \mathcal{Z}^{J}=2 m R
$$

which takes arbitrary real values $m$. The parameter $m$ has the dimension of a mass. Indeed, we will relate it to the mass of a particle propagating in the $A d S_{5}$ background. Before discussing this we contrast these constraints to the conformal space. In that case there is only one twistor and the quadratic invariant

$$
\mathcal{Z Z}=\mathcal{Z}_{0} \mathcal{Z}_{0}
$$

necessarily vanishes due to (16). This equation defines a surface in twistor space, which is related to the phase space of a massless particle [3].

The first order (or phase space) action of a 'massive' particle in $A d S_{5}$ is given by

$$
S=\int d \tau\left[\tilde{P}_{\tilde{m}} \partial_{\tau} \tilde{x}^{\tilde{m}}-\frac{e}{2}\left(\tilde{P}_{\tilde{m}} \tilde{g}^{\tilde{m} \tilde{n}} \tilde{P}_{\tilde{n}}+m^{2}\right)\right] .
$$

The momenta $\tilde{P}_{\tilde{m}}=\left(P_{m}, P_{\rho}\right)$ are conjugate to $\tilde{x}^{\tilde{m}}=\left(x^{m}, \rho\right)$ and $\tilde{g}^{\tilde{m} \tilde{n}}$ is the inverse $A d S_{5}$-metric (3). Translating this into two-component notation yields

$$
S=-\int d \tau\left[\frac{1}{2} P_{\alpha \dot{\alpha}} \dot{x}^{\dot{\alpha} \alpha}-P_{\rho} \dot{\rho}-\frac{e}{2 R^{2}}\left(\frac{1}{2 \rho^{2}} P_{\alpha \dot{\alpha}} P^{\dot{\alpha} \alpha}-\rho^{2} P_{\rho}^{2}-m^{2} R^{2}\right)\right],
$$

where

$$
P_{\alpha \dot{\alpha}}=R P_{m}\left(\sigma^{m}\right)_{\alpha \dot{\alpha}} .
$$


The worldline einbein $e(\tau)$ is a Lagrange multiplier for the mass-shell constraint

$$
\frac{1}{2 \rho^{2}} P_{\alpha \dot{\alpha}} P^{\dot{\alpha} \alpha}-\rho^{2} P_{\rho}^{2}=m^{2} R^{2} .
$$

We rewrite the momenta in terms of the twistor variables $\lambda_{\alpha}^{I}$ introduced above by setting

$$
\begin{aligned}
P_{\alpha \dot{\alpha}} & =2 \lambda_{\alpha}^{I} \bar{\lambda}_{\dot{\alpha}}^{I}, \\
P_{\rho} & =-\frac{i}{2 \rho^{2}}\left(\varepsilon^{\alpha \beta} \epsilon^{I J} \lambda_{\alpha}^{I} \lambda_{\beta}^{J}-\text { c.c. }\right),
\end{aligned}
$$

and the second pair of twistor components $\mu^{I}$ are introduced as in (29). It follows straightforwardly that

$$
\frac{1}{2 \rho^{2}} P_{\alpha \dot{\alpha}} P^{\dot{\alpha} \alpha}-\rho^{2} P_{\rho}^{2}=\left(\frac{1}{2 \rho}\left(\varepsilon^{\alpha \beta} \epsilon^{I J} \lambda_{\alpha}^{I} \lambda_{\beta}^{J}+\text { c.c. }\right)\right)^{2}=\frac{1}{4}\left(\overline{\mathcal{Z}}^{I} \delta^{I J} \mathcal{Z}^{J}\right)^{2} .
$$

The constraint (41) forces this to equal $m^{2}$ and therefore we relate the invariant $\overline{\mathcal{Z}}^{I} \delta^{I J} \mathcal{Z}^{J}$ to the mass $m$ as in (36). In other words (36) is equivalent to the mass-shell constraint. It turns out that, like for the massless particle in $1+3$ dimensions [6], the action for an on-shell particle (41) can be rewritten entirely in twistor variables,

$$
S=-\int d \tau\left(\frac{1}{2} P_{\dot{\alpha} \alpha} \partial_{\tau} x^{\dot{\alpha} \alpha}-P_{\rho} \partial_{\tau} \rho\right)=-i \int d \tau \overline{\mathcal{Z}}^{I} \partial_{\tau} \mathcal{Z}^{I},
$$

where the twistor pair is subject to the four real constraints (35) and (36)

$$
\phi_{a}=\overline{\mathcal{Z}}^{I} t_{a}^{I J} \mathcal{Z}^{J}-2 \delta_{a}{ }^{0} m R .
$$

If we wish to elevate the twistors to independent variables, the constraints (45) have to be imposed via Lagrange multipliers $u^{a}$ and the total action becomes

$$
S=-i \int d \tau\left(\overline{\mathcal{Z}}^{I} \partial_{\tau} \mathcal{Z}^{I}-i u^{a} \phi_{a}\right) .
$$

The appearence of the Lagrange multiplier terms generates gauge symmetries which act on the fields as

$$
\begin{aligned}
\delta \mathcal{Z}^{I} & =i \xi^{a}(\tau) t_{a}^{I J} \mathcal{Z}^{J}, \\
\delta u^{0} & =\partial_{\tau} \xi^{0}(\tau), \\
\delta u^{i} & =\partial_{\tau} \xi^{i}(\tau)+2 i \varepsilon^{i j k} \xi^{j}(\tau) u^{k},
\end{aligned}
$$

where $\xi^{a}(\tau)$ are 4 local parameters and form an $U(1) \times S U(2)$ algebra. Therefore, we can rewrite the action (46) as a 1-dimensional gauge theory action

$$
S=-i \int d \tau\left(\overline{\mathcal{Z}}^{I} D_{\tau} \mathcal{Z}^{I}+2 i u^{0} m R\right)
$$


where

$$
D_{\tau} \mathcal{Z}^{I}=\left(\delta^{I J} \partial_{\tau}-i u^{a} t_{a}^{I J}\right) \mathcal{Z}^{J}
$$

and the Lagrange multipliers act as gauge fields.

We have shown that the construction of twistors, realizing the conformal symmetry $S U(2,2)$ of 4-dimensional Minkowski space linearly, can be generalized to include the radial coordinate of the $A d S_{5}$ space. To do so, we employed the coset descriptions of the conformal space and the $A d S_{5}$ space, and we had to extend the twistor construction to a pair of twistors subject to important constraints. In essence, we built a bridge between the twistor formulation of $S U(2,2)$, and the description of $A d S_{5}$ as a hyperboloid in $(2,4)$ dimensions. In the latter, the coordinates of the 6-dimensional embedding space also realize the conformal symmetry $G=S O(2,4) \sim S U(2,2)$ linearly, although in the fundamental of $S O(2,4)$. The generalized twistors realize the symmetry linearly in the spinor representation of $S O(2,4)$ which is equivalent to the fundamental representation of $S U(2,2)$. One advantage of our procedure is that it seems to have a straightforward generalization to the supersymmetric case $S U(2,2 \mid \mathcal{N})$, i.e. to find a twistor realization of the $A d S_{5} \times S^{5}$ superspace. All one needs to do is to construct the supercoset representative and find the proper constraint on $\mathcal{Z}_{0}$. This will essentially be the same constraints as for the bosonic space since the compensating transformation in the supersymmetric case is also purely bosonic. In that way one could find the supersymmetric generalization of defining $A d S_{5} \times S^{5}$ as a 10-dimensional hypersurface in 12 dimensions.

We have also shown that these twistors appear naturally as the fundamental variables of a massive particle propagating in $A d S_{5}$. It would be interesting to investigate whether we can extent the superparticle action to a simple supertwistor one as well.

The most exciting aspect of our result however is the simplicity of the action (48). The quantization of this action will be considered in an accompanying paper [5]. It might pave the way to a simple quantization of the string on $A d S_{5}$. One would hope that (44) generalizes to the string action in the same straightforward way it did in flat space, viewed as the boundary limit of $A d S_{5}$ ([6] vs. [1]). If this is the case then the string spectrum constructed in [1] from a stringy toy world-sheet model should indeed be the spectrum of strings on $A d S_{5} \times S^{5}$.

Acknowledgements: We enjoyed very useful discussions with Renata Kallosh. The work of P.C. was supported by the European Commission TMR program ERBFMRXCT96-0045. J.R. and Y.Z. were supported in part by NSF grant PHY-9870115. 


\section{Spinor notation}

Here we collect some useful formulae and fix notation. We define the $\sigma$-matrices as

$$
\sigma_{m}=\left\{\mathbb{1}, \hat{\sigma}_{i}\right\}, \quad \bar{\sigma}_{m}=\left\{\mathbb{1},-\hat{\sigma}_{i}\right\}, \quad \sigma_{m n}=\sigma_{[m} \bar{\sigma}_{n]}, \quad \bar{\sigma}_{m n}=\bar{\sigma}_{[m} \sigma_{n]},
$$

where $\hat{\sigma}_{i}$ are the conventional Pauli-matrices satisfying $\hat{\sigma}_{1} \hat{\sigma}_{2}=i \hat{\sigma}_{3}$. They have the index structure

$$
\left(\sigma_{m}\right)_{\alpha \dot{\alpha}}, \quad\left(\bar{\sigma}_{m}\right)^{\dot{\alpha} \alpha}, \quad\left(\sigma_{m n}\right)_{\alpha}^{\beta}, \quad\left(\bar{\sigma}_{m n}\right)_{\dot{\beta}}^{\dot{\alpha}} .
$$

Undotted indices are always contracted NW-SE, while dotted indices are contracted SW-NE. Indices are contracted with an $\varepsilon$-tensor, defined by

$$
\varepsilon_{12}=-\varepsilon_{\dot{1} \dot{2}}=\varepsilon^{12}=-\varepsilon^{\dot{i} \dot{2}}=1, \quad \varepsilon^{\alpha \gamma} \varepsilon_{\beta \gamma}=\delta_{\beta}^{\alpha}, \quad \varepsilon_{\dot{\alpha} \dot{\gamma}} \varepsilon^{\dot{\beta} \dot{\gamma}}=\delta_{\dot{\alpha}}^{\dot{\beta}} .
$$

This implies the relationships

$$
\left(\sigma_{m}\right)_{\alpha \dot{\alpha}}=\left(\sigma_{m}^{T}\right)_{\dot{\alpha} \alpha}=\varepsilon_{\dot{\alpha} \dot{\beta}}\left(\bar{\sigma}_{m}\right)^{\dot{\beta} \beta} \varepsilon_{\beta \alpha}, \quad\left(\bar{\sigma}_{m}\right)^{\dot{\alpha} \alpha}=\left(\bar{\sigma}_{m}^{T}\right)^{\alpha \dot{\alpha}}=\varepsilon^{\alpha \beta}\left(\sigma_{m}\right)_{\beta \dot{\beta}} \varepsilon^{\dot{\beta} \dot{\alpha}}
$$

We also have the identities

$$
\sigma_{(m} \bar{\sigma}_{n)}=\bar{\sigma}_{(m} \sigma_{n)}=-\eta_{m n} \mathbb{1}
$$

where $\eta=\operatorname{diag}(-,+,+,+)$. To translate between spinor notation and vector notation we associate a real 4-dimensional vector $X^{m}$ to a hermitian matrix

$$
X^{\dot{\alpha} \alpha}=X^{m}\left(\bar{\sigma}_{m}\right)^{\dot{\alpha} \alpha} \quad \text { and } \quad X_{\alpha \dot{\alpha}}=X^{m}\left(\sigma_{m}\right)_{\alpha \dot{\alpha}} \quad \rightarrow \quad X_{\alpha \dot{\alpha}}=\varepsilon_{\dot{\alpha} \dot{\beta}} X^{\dot{\beta} \beta} \varepsilon_{\beta \alpha} .
$$

In the same way we translate a real anti-symmetric tensor $T^{m n}$ to the pair of chiralantichiral matrices

$$
T_{\alpha}{ }^{\beta}=\frac{1}{4} T^{m n}\left(\sigma_{m n}\right)_{\alpha}{ }^{\beta} \quad \text { and } \quad \bar{T}_{\dot{\beta}}^{\dot{\alpha}}=-\frac{1}{4} T^{m n}\left(\bar{\sigma}_{m n}\right)_{\dot{\beta}}^{\dot{\alpha}}
$$

which are related by hermitian conjugation.

The 5 -dimensional $\gamma$-matrices are constructed out of $\sigma, \bar{\sigma}$ by

$$
\gamma_{m}=i\left(\begin{array}{cc}
0 & \sigma_{m} \\
\bar{\sigma}_{m} & 0
\end{array}\right), \quad \gamma_{\rho} \equiv \gamma_{5}=i \gamma_{0} \gamma_{1} \gamma_{2} \gamma_{3}=\left(\begin{array}{cc}
-\mathbb{1} & 0 \\
0 & \mathbb{1}
\end{array}\right)
$$

\section{References}

[1] P. Claus, M. Gunaydin, R. Kallosh, J. Rahmfeld and Y. Zunger, Supertwistors as Quarks of $S U(2,2 \mid 4)$, J. High Energy Phys. 05 (1999) 019, hep-th/9905111. 
[2] R. Penrose, Twistor algebra, J. Math. Phys. 8 (1967) 345; R. Penrose and M. A. H. MacCallum, Twistor Theory: An Approach To The Quantisation Of Fields And Space-Time, Phys. Rept. 6 (1972) 241.

[3] A. Ferber, Supertwistors and Conformal Supersymmetry, Nucl. Phys. B132 (1978) 55.

[4] J. Maldacena, The Large N Limit of Superconformal Field Theories and Supergravity, Adv. Theor. Math. Phys. 2 (1998) 231, hep-th/9711200.

[5] P. Claus, R. Kallosh and J. Rahmfeld, BRST Quantization of a Particle in AdS $S_{5}$ Space, in preparation.

[6] P.K. Townsend, Supertwistor Formulation of the Spinning Particle, Phys. Lett. B261, 65 (1991).

[7] P. Claus and R. Kallosh, Superisometries of the $A d S \times S$ Superspace, J. High Energy Phys. 03 (1999) 014, hep-th/9812087. 